Revue d'histoire de l'Amérique française

REVUE D.HISTOIRE DE L'AMÉRIQUE FRANÇAISE

\title{
L’Amérindien victime de l'incompétence des historiens
}

\section{James S. Pritchard}

Volume 41, numéro 1, été 1987

URI : https://id.erudit.org/iderudit/304523ar

DOI : https://doi.org/10.7202/304523ar

Aller au sommaire du numéro

Éditeur(s)

Institut d'histoire de l'Amérique française

ISSN

0035-2357 (imprimé)

1492-1383 (numérique)

Découvrir la revue

Citer cette note

Pritchard, J. S. (1987). L’Amérindien victime de l'incompétence des historiens. Revue d'histoire de l'Amérique française, 41(1), 63-70.

https://doi.org/10.7202/304523ar d'utilisation que vous pouvez consulter en ligne.

https://apropos.erudit.org/fr/usagers/politique-dutilisation/ 


\title{
NOTES CRITIQUES \\ L'amérindien victime de l'incompétence des historiens ${ }^{1}$
}

\author{
JAMES S. PRITCHARD \\ Département d'histoire \\ Université Queen's
}

Parmi les sciences humaines ayant contribué à une meilleure compréhension de l'histoire, les sciences économiques ont longtemps tenu la vedette avant d'être rejointes, il y a une vingtaine d'années, par d'autres disciplines. Les géographes, par exemple, ont clairement démontré la pertinence de leurs perspectives et plusieurs de leurs travaux ont amené des réinterprétations significatives de l'histoire canadienne $^{2}$. Les mathématiciens et statisticiens ont également transformé la démographie historique pour en faire la base d'une nouvelle histoire sociale ${ }^{3}$. Il y a une dizaine d'années, Bruce Trigger, un archéologue devenu anthropologue, a fait entrer l'ethnohistoire comme l'un des principaux thèmes dans le discours historique ${ }^{4}$ et son étude remarquable du peuple huron en fit le spécialiste prééminent dans le domaine. Toutefois, malgré l'utilité de cette oeuvre, sa plus récente contribution, Natives and Newcomers, comporte de graves erreurs qui réveillent les vieilles interrogations concernant l'efficacité des études interdisciplinaires.

Ce livre ${ }^{5}$ a été généralement bien reçu. D'après la publicité sur la jaquette, le Toronto Star le juge «a landmark, a milestone, epochal»;

\footnotetext{
I Je remercie mon collègue $F$. T. Butts qui a bien voulu commenter une version préliminaire de cet article.

Richard Colebrook Harris, The Seigneurial System in Early Canada: a Geographical Study (Québec, Les Presses de l'Université Laval, 1966 [édition révisée, Montréal et Kingston, McGill-Queen's University Press, 1984]); Andrew Hill Clark, Acadia: the Geography of Early Nova Scotia to 1760 (Madison, University of Wisconsin Press, 1968); Conrad Heidenreich, Huronia: a History and Geography of the Huron Indians, 1600-1650 (Toronto, McClelland \& Stewart, 1971); et Arthur J. Ray, Indians in the Fur Trade (Toronto, University of Toronto Press, 1974). Hubert Charbonneau, Vie et mort de nos ancêtres: étude démographique (Montréal, Les Presses de l'Université de Montréal, 1975).

${ }^{4}$ Bruce Graham Trigger, The Children of Aataentsic: a History of the Huron People to 1660 (Montréal et Kingston, McGill-Queen's University Press, 1976), 2 volumes.

5 Bruce Graham Trigger, Natives and Newcomers: Canada's «Heroic Age» Reconsidered (Montréal et Kingston, McGill-Queen's University Press, 1985).
} 
le Globe and Mail le considère «essentiel» pour tous ceux qui s'intéressent à l'histoire canadienne; tandis que Quill and Quire estime qu'il s'agit d'une oeuvre «héroïque». Le collaborateur du Canadian Geographic complimente Trigger pour son usage des techniques de l'ethnohistoire et insiste sur les perspectives «révisionistes», aspect repris dans d'autres comptes rendus ${ }^{6}$. Mais le compte rendu le plus enthousiaste va beaucoup plus loin; d'après Boyce Richardson, le livre ne constitue pas seulement une oeuvre d'une érudition extraordinaire, mais son auteur mérite de prendre place auprès de Harold Innis, Northrop Frye et Marshall McLuhan au panthéon des grands esprits canadiens?

Les éloges de la part des universitaires sont moins lyriques, mais tout aussi fréquents et directs. William N. Fenton, doyen des spécialistes sur l'iroquoisie, considère Natives and Newcomers «the most important book on the historiography, archaeology and ethnohistory of North America to appear in a decade» (jaquette). John Dickinson, dans The American Historical Review, estime que malgré quelques défauts, l'érudition est fondamentalement bonne et conclut que l'oeuvre constitue «a very useful contribution to early Canadian history» ${ }^{8}$. Le compte rendu de Francis Jennings dans The Canadian Historical Review est tout à fait incomplet, n'étant qu'un ramassis d'impressions diverses, mais comme les autres auteurs il insiste sur l'importance des conclusions révisionistes de Trigger 9 . Les seules réserves sérieuses sont de la plume de William J. Eccles qui, néanmoins, félicite l'auteur d'avoir rédigé «the most cogent treatment of the [Indian image in Canadian history] yet to appear». Le reste, cependant, est décrit comme étant «rather heavy going», «stale», rempli d'anachronismes et «a maze of contradictions» ${ }^{10}$. Toutes ces appréciations passent à côté du problème et plusieurs invitent à une lecture sans esprit critique de l'oeuvre de Trigger. Natives and Newcomers n'offre pas une réinterprétation simple de l'histoire canadienne. Trigger ne comprend pas l'essence du métier d'historien et son livre constitue une incrimination de l'historiographie canadienne et de la méthodologie des historiens.

Dans son dernier livre, Trigger affirme que les historiens ont tellement mal écrit l'histoire du Canada que les savants venus d'autres disciplines, notamment d'archéologie et d'anthropologie sociale, doivent refaire le travail à leur place. D'après lui, les historiens sont des sots qui, autrefois, débattaient la question de savoir s'il fallait faire débuter l'histoire du Canada en l'an mil, en 1497 ou en 1534. Pour des raisons

\footnotetext{
6 Canadian Geographic (April-May 1986): 78; voir aussi Canadian Book Review Annual (1985): 368, et Queen's Quarterly (Summer 1986): 404-406.

7 Saturday Night (July 1986): 49.

8 The American Historical Review, 91,5 (December 1986): 1294-1295.

9 The Canadian Historical Review, 67,2 (June 1986): 249-251.

10 William and Mary Quarterly, 3rd Series, 43,3 (July 1986): 481-482.
} 
inconnues, «historians most often study whole societies rather than communities or families» et ils ont «a continuing tendency to explain behaviour in terms of different national characters». Les historiens sont également coupables de «naturally pay most attention to interest groups that are of historical, rather than social or cultural, significance» ${ }^{1 P}$. Bien sûr, aucune preuve n'est offerte pour appuyer ces généralisations concernant les historiens. La réinterprétation des rapports entre Européens et Iroquoiens dans l'est de l'Amérique du Nord n'est, en fait, qu'un prétexte pour critiquer les historiens; sa prétention de modifier d'une manière radicale notre compréhension de la colonisation du Canada jusqu'en 1663 en tenant compte des buts et aspirations des autochtones est secondaire ${ }^{12}$. Natives and Newcomers constitue donc moins une contribution à la littérature sur les rapports franco-amérindiens qu'une oeuvre polémique dirigée contre les historiens. Ce n'est pas une oeuvre d'histoire et, malheureusement, ce n'est pas de la bonne polémique car pour avoir du succès ce genre doit être bien fondé dans des observations empiriques et soutenu en tenant compte des écoles différentes. Or, Natives and Newcomers ne rencontre aucun de ces critères.

Le livre est divisé en six chapitres. Les deux premiers prétendent que les méthodes de l'archéologie et de l'anthropologie permettent une compréhension objective bien supérieure à tout ce que peut offrir l'histoire traditionnelle. Les chapitres trois, quatre et cinq comprennent la réinterprétation de l' «âge héroïque». Dans son dernier chapitre, Trigger présente sa conclusion principale: le peuplement de la Nouvelle-France n'a pas eu lieu malgré la traite des fourrures, mais résulte directement de cette activité et des alliances amérindiennes.

Invectiver Francis Parkman et ses semblables ne sert plus à rien sinon à ennuyer le lecteur, et voilà le contenu du premier chapitre. Le fait que Trigger condamne les historiens d'autrefois de ne pas écrire l'histoire comme il le voudrait illustre clairement son incapacité à comprendre ce que faisaient les historiens de cette époque aussi bien que ceux d'aujourd'hui. Toute sa discussion est prise hors contexte et présentée sans égard à la tradition intellectuelle occidentale. Les stéréotypes des peuples autochtones, par exemple, sont perçus comme des barrières à la compréhension historique plutôt que faisant partie intégrante d'interprétations différentes en évolution. Trigger refuse le concept que l'histoire est fondée sur l'accumulation de connaissances. D'après lui, l'histoire permet d'arriver à une compréhension objective et n'a donc pas besoin du travail des précurseurs; il suffit de raisonner correctement. L'idée que la perspicacité de certains historiens contem-

\footnotetext{
11 Trigger, op. cit., 5, 6, 170, 299-300.

12 Ibid., 226.
} 
porains est fondée sur le travail de ceux qui les ont précédés ne lui effleure pas l'esprit. L'histoire traditionnelle «gives rise only to spectacular but partial interpretations of native behaviour». Seule une compréhension anthropologique détaillée des cultures autochtones «allows a reasonable balance to be struck between the distinctive qualities of native cultures and the equally important characteristics shared by all human beings» ${ }^{13}$. Pour le «social scientist» qu'est Trigger, il ne peut exister qu'une interprétation, celle qui est objectivement vraie.

Dans le deuxième chapitre, Trigger veut démontrer que l'archéologie donne à l'historien une méthode nouvelle et plus efficace pour atteindre à la vérité: «archaeology is able to produce a history of peoples rather than a mere chronicle of objects. ${ }^{14}$ Le chapitre se divise en deux parties. En premier lieu, on retrouve une synthèse apparemment complète et à jour du développement de l'archéologie nord-américaine avec des références particulières aux Iroquois qui pourra «être utile aux historiens de l'archéologie. En second lieu, Trigger présente l'état actuel des interprétations archéologiques du développement social des Iroquoiens avant l'arrivée des Blancs. En tentant de démontrer que le 14e siècle est témoin de transformations culturelles fondamentales dont un accroissement de l'activité guerrière, Trigger veut prouver que des changements importants pouvaient être causés par autre chose que l'arrivée des Européens. Malheureusement son argument souffre des mêmes problèmes que ceux du chapitre précédent. Même si cette interprétation est fondée, elle n'invalide pas les opinions - Trigger les qualifie de révisionisme gratuit - de ceux qui perçoivent les transformations des $16 \mathrm{e}$ et $17 \mathrm{e}$ siècles comme des conséquences de la présence européenne.

Les trois chapitres suivants comportent l'essentiel de la nouvelle interprétation de l'âge héroïque mise de l'avant par Trigger. Après avoir brossé un bref tableau des activités européennes le long de la côte atlantique au 16e siècle, tableau truffé de spéculations oisives sur la circulation d'articles de traite chez les Amérindiens, Trigger présente une ethnohistoire des Iroquoiens pendant les sept premières décennies du $17 \mathrm{e}$ siècle. Le contenu de ces chapitres reprend en le condensant à l'extrême son Children of Aataentsic. Malgré les prétentions méthodologiques de l'auteur, la chronologie adoptée est empruntée aux historiens et n'est pas fonction de son approche. Qui plus est, ces chapitres sont bourrés de spéculations; des termes tels «il se peut», «peut avoir», «on peut penser que», «probablement», «on peut déduire que», «semble avoir», ainsi que l'emploi du conditionnel font en sorte que le lecteur n'est sûr de rien au terme de cette réinterprétation et même le caractère polémique en souffre. Si l'ouvrage précédent était indubitablement un

13 Ibid., 46, 299

14 Ibid., 110. 
chef-d'oeuvre, ce résumé ne lui rend pas justice et lui enlève les qualités littéraires et d'imagination érudite qui lui étaient propres.

Dans le troisième chapitre, Trigger insiste sur l'existence d'une traite des fourrures avant Cartier, sans cependant en offrir de preuves ${ }^{15}$. Sa méthode est véritablement douteuse. Au départ, il fonde sa crédibilité en tant que spécialiste en sciences humaines en s'attaquant à des hommes de paille: on a droit à des spéculations bizarres, c'est le moins qu'on puisse dire, sur des visiteurs israélites, romains, hindous, irlandais, scandinaves ou portugais en Amérique ${ }^{16}$. Ensuite, il ignore les travaux de plusieurs historiens de renom car, d'après lui, rien de valable n'a paru pendant le demi-siècle qui sépare les travaux de H. P. Biggar et W. F. Ganong de ceux de D. B. Quinn, Selma Barkham et J. A. Tuck (qui est archéologue bien entendu!) dans les années 1970. Les CharlesAndré Julien, Marcel Destombes, Suzanne Lussagnet et Marcel Trudel ne méritent même pas une référence ${ }^{17}$. Les suppositions et généralisations sans but apparent pleuvent. Dans un paragraphe de cinq phrases, par exemple, on retrouve en ordre: "perhaps» "possibly», «appear to have been», «appear to have ranged» et «perhaps» ${ }^{18}$. Enfin, Trigger utilise ses sources d'une manière très sélective. Il cite $\mathrm{D}$. B. Quinn pour appuyer sa prétention que les baleiniers étaient déjà actifs dans la baie des Chasteaux en 1529, mais en vérifiant Quinn on découvre que celuici n'a jamais fait une affirmation aussi catégorique ${ }^{19}$. Les lecteurs devraient, en effet, vérifier les sources de Trigger pour comparer sa «compréhension objective» à leur propre interprétation des événements de cette époque.

L'usage que Trigger fait des concepts est également curieux. Une bonne partie du quatrième chapitre constitue une étude systématique du concept de groupe d'intérêt. Il a bien raison d'inciter les historiens à faire un effort pour comprendre les valeurs culturelles de l'observateur et de celui qu'on observe et de suggérer que, dans le cas de peuples analphabètes, le concept de groupe d'intérêt dont les membres partagent des buts communs et se soutiennent permet d'interpréter leurs actions $^{20}$. Trigger semble se servir efficacement de ce concept lorsqu'il analyse les actions des autochtones, mais son traitement des comportements européens est moins heureux. Sa détermination à démontrer que Champlain a mal agi dans ses rapports avec les autochtones illustre à merveille le mauvais usage que Trigger fait de ce concept. Il infère la motivation personnelle de l'individu à partir de généralisations hâtives

\footnotetext{
15 Ibid., 127-128.

16 Ibid., 118-119.

17 Ibid., 111.

8 Ibid., 122.

19 Ibid., 128.

Ibid., 168-169.
} 
et discutables tirées de sources secondaires sur les comportements collectifs $^{21}$. Ses commentaires sur les groupes d'intérêt français sont simplistes à l'absurde. Tous les commerçants appartiennent à un groupe, tous les missionnaires à un autre, et en considérant Champlain comme un groupe d'intérêt, il va jusqu'à lui enlever son individualité ${ }^{22}$. C'est peut-être son but car Trigger affirme que la traite forme la base de tous les rapports entre Français et Amérindiens ${ }^{23}$. Cette prétention constitue la clé de la réinterprétation de Trigger.

Dès lors, il est évident que Trigger aura du mal à situer les missionnaires dans ce contexte; son interprétation de la présence jésuite en Huronie au chapitre cinq a en effet de quoi surprendre. Après 225 pages consacrées à l'obscurantisme des historiens, à l'avarice des Français, à la sagesse des Hurons et aux fondements économiques des rapports inter-ethniques, il doit rendre compte de l'arrivée de pauvres missionnaires dans un pays où ils n'étaient pas les bienvenus. Pour lui l'explication est l'évidence même. Tout d'un coup en 1634, pendant qu'ils se remettaient de la prise de Québec par des corsaires anglais, les Français, incompétents jusqu'alors, réussissent à déjouer la perspicacité huronne en leur imposant les missionnaires par supercherie ${ }^{24}$. On est en droit de se demander à quoi attribuer cette naïveté des Hurons.

En ce qui concerne le rôle des épidémies sur les populations autochtones, Trigger laisse le lecteur sur sa faim. Deux interprétations des taux de mortalité dans l'est de l'Amérique du Nord sont imposées: l'une prétend que des épidémies massives au 16e siècle décimèrent la population amérindienne; l'autre considère que les maladies n'affectèrent les autochtones qu'à partir de la deuxième décennie du $17 \mathrm{e}$ siècle ${ }^{25}$. Tout en présentant ces interprétations, Trigger oublie de mentionner qu'il s'agit d'hypothèses qu'on ne pourra vraisemblablement jamais vérifier. Ensuite, Trigger trahit la pensée de plusieurs auteurs en confondant les maladies chroniques chez les populations pré-colombiennes, ce que personne ne nie, avec les contagions virales et bactériennes importées en Amérique. Ce qui lui permet de supposer, sans fondement documentaire, que «there were probably some outbreaks of indigenous epidemic diseases as well» ${ }^{26}$.

Avec un illogisme étonnant, Trigger nie que la mortalité massive chez les Hurons ait eu des effets importants sur la traite. D'abord, il affirme sans preuves que le nombre de peaux offertes aux Français ne

Ibid., 317-319.

Ibid., 173-174, 198-199, 200-203.

Ibid., 224, 331.

Ibid., 229.

Ibid., 237-238.

Ibid., 237-238 et 244. 
diminua point après 1640 et qu'il aurait même augmenté. Ensuite, il prétend que les chasseurs des Grands Lacs ne recevaient que peu d'articles de traite. Enfin, il suppose que l'effort requis pour doubler ou tripler le nombre de peaux rapportées par chasseur était minime. Tout ceci pour conclure que : «As a result many more European goods were accessible to each family than had been previously. ${ }^{27}$ De fait, on ne peut rien tirer de deux affirmations sans fondement, d'une conjecture et d'une supposition sauf, peut-être, de la confusion.

Depuis vingt ans, plusieurs travaux, y compris ceux de Trigger, ont renversé l'historiographie traditionnelle des débuts de la NouvelleFrance en insistant sur le rôle joué par la culture amérindienne dans l'élaboration des réseaux de traite. Trigger a raison de souligner que la compréhension du comportement huron requiert une sensibilité supérieure à celle dont les historiens ont fait preuve. Il est donc ironique que les études de Trigger ne cernent pas d'une manière adéquate la culture et l'idéologie huronnes. Sa discussion du monde mythique huron est laconique $^{28}$ et il se contente d'affirmer que leurs croyances étaient dynamiques et résistantes aux chocs ${ }^{29}$. Il semble réticent à admettre qu'ils avaient un univers mental propre.

La raison de cette omission vient simplement de la perspective matérialiste de Trigger. Il reconnaît les ambitions huronnes et insiste sur leur importance mais il les confine aux rapports sociaux, politiques et économiques. Ainsi il rejette les interprétations de Clifford Geertz, de Marshall Sahlins et de Calvin Martin qui affirment que c'est à travers la culture et l'idéologie que les membres d'une société donnent un sens à leurs rapports interpersonnels et avec l'environnement. Les lacunes de l'interprétation matérialiste de Trigger sautent aux yeux lorsqu'il traite des missionnaires jésuites. Il est tout aussi incapable de comprendre les motivations spirituelles des Français que celles des Hurons. Ainsi s'explique son rejet de l'oeuvre du père Lucien Campeau, à qui on peut pardonner de croire que la volonté humaine n'est pas uniquement dirigée par l'intérêt matériel. Mais si Trigger nie que certaines facettes du monde huron puisse demeurer incompréhensibles à des esprits cartésiens, s'il veut à tout prix démontrer que le comportement huron est logique, rationnel et facile à expliquer, c'est que reconnaitre le contraire démolirait sa prétention que l'archéologie et l'anthropologie sociale permettent une meilleure compréhension objective du développement historique du Canada que l'histoire. Sa démonstration est loin d'être convaincante et on ne voit pas comment l'anthropologie pourrait aider à «understand better the internal dynamics of French colonization» ${ }^{30}$. 
Dans Natives and Newcomers, Bruce Trigger démontre qu'il ne comprend pas ce que font les historiens ni comment leur métier diffère des autres activités scientifiques. D'après le grand poète mexicain, Octavio $\mathrm{Paz}$, les poètes, les scientifiques et les historiens nous révèlent l'envers de la médaille, le visage caché de la langue, de la nature ou du passé. Mais, se demande-t-il, si le poète produit des métaphores, le scientifique des lois naturelles, que produit l'historien? Paz répond que l'historien se place entre le poète qui aspire à créer une image unique et le scientifique qui veut réduire l'individu à une loi. Comme le poète, il examine des cas individuels et, comme le scientifique, il cherche à lire dans les événements des tendances. Sa recherche de cohérence l'apparente au scientifique, mais la découverte de la cohérence prend la forme d'une fable poétique. Comme la science, l'histoire est découverte; comme la poésie, elle est une oeuvre de création. Toutefois, l'histoire ne découvre pas des mondes nouveaux comme la science, et ne les invente pas comme la poésie; elle reconstruit le monde du passé. L'histoire n'est pas un savoir, stricto sensu, c'est une sagesse. Voilà la véritable tradition historiographique de l'Occident, qu'elle soit issue de saint Augustin ou de Karl Marx ${ }^{31}$. Ne pas reconnaître le caractère particulier de l'histoire condamne Natives and Newcomers à échouer dans sa tentative de démontrer que l'archéologie et l'anthropologie possèdent un cadre conceptuel et méthodologique essentiel pour réécrire l'histoire canadienne.

Traduction : John A. Dickinson

31 Octavio Paz, «Forward» dans Jacques La Faye, Quetzalcoatl and Guadaloupe: the Formation of Mexican National Consciousness, 1531-1813, traduit par Benjamin Keen (Chicago, University of Chicago Press, 1976), ix-x. 